\title{
Objectively Measured Commute Distance: Associations with Actual Travel Modes and Perceptions to Place of Work or Study in Auckland, New Zealand
}

\author{
Hannah M. Badland, Grant M. Schofield, \\ and Philip J. Schluter
}

\begin{abstract}
Background: Little is known about the relationships between objectively measured commute distance with actual and perceived transport-related physical activity (TPA) engagement. Methods: A telephone survey assessed travel behaviors to place of work/study within an adult sample $(n=772)$ residing in New Zealand. Result: Overall, $50 \%$ of respondents perceived they could, and $10 \%$ of the sample actually did, use TPA modes to commute to their occupation for distances less than $5 \mathrm{~km}$. Differences between TPA perceptions and engagement existed for all distance classifications, and prevalence declined as distances increased. Conclusions: Differences between TPA engagement and perceptions were evident. Actual and perceived TPA engagement levels declined as commute distance increased.
\end{abstract}

Key Words: Geographical information systems; physical activity; transport; occupation; adults

Motorized transport is now the dominant form of travel for the majority of developed countries, contributing in part to increased traffic congestion, air pollution, and diminishing community cohesion. ${ }^{1}$ Simultaneously, physical inactivity is a major public health concern for these countries. ${ }^{2}$ One potential solution to minimize problems associated with automobile reliance and physical inactivity is to promote transport-related physical activity (TPA) modes for traveling short distances. Research has shown individuals who engage in TPA modes have shorter self-reported network distances to their occupation than those who commute by automobile. ${ }^{3}$ Although commute distance plays a pivotal role in transport modal choice, perceptions should also be considered. An Australian study reported that time travel perception was the most common barrier to TPA/transit engagement to university for employees and students, followed by perceived commute distance. ${ }^{4}$

The authors are with the Centre for Physical Activity and Nutrition Research, Faculty of Health and Environmental Sciences, AUT University, Auckland, New Zealand. 
This study builds the commute distance evidence by using geographical information systems (GIS) route measures, actual travel mode, and TPA perceptions in relation to accessing place of work/study for the first time using an adult regional representative sample.

\section{Methods}

\section{Study Sample}

The Active Friendly Environments Survey (AFES) was implemented in a representative adult sample ( $\geq 16 \mathrm{y}$ of age) of North Shore City, (Auckland, New Zealand) residents in 2005. Participants were drawn randomly from the electronic telephone white pages and contacted through computer assisted telephone interview procedures. Overall, 2000 respondents were recruited (31\% response rate). Consent was provided prior to participation and the Auckland University of Technology Ethics Committee approved the study.

\section{AFE Travel Mode Measures}

The TPA component reported typical travel modes for commuting from residence to occupation. Respondents were asked "How do you usually get to and from your place of work/study?" Answers were collapsed into three travel mode categories: motorized, TPA, and transit/combined.

\section{AFE TPA Perceptions}

Participants reported whether they recognized they could engage in TPA modes to access their occupation from their residence by responding yes or no to the question: "Do you think that you could access your place of work/study by traveling on foot or cycling?"

\section{Commute Distance}

Commute distances between residence and place of work/study were calculated using ArcView version 9.1 OD Cost Matrix function GIS software. Residential and place of work/study addresses were obtained directly from respondents and geocoded. The shortest street network distance between the points was calculated in kilometers $(\mathrm{km})$ for every resident. Distances were collapsed into four categories.

\section{Statistical Analysis}

Proportions and exact associated confidence intervals were made based on the binomial assumption. Crude and adjusted relative risk estimates were made using binary logistic regression analyses. Statistical comparisons were made using SPSS version 11.5 and Stata version 8.0. 


\section{Results}

Overall, 772 respondents from the sample were eligible for analysis as they resided and engaged in an occupation within North Shore City. Motorized travel was the dominant mode of transport to access place of work/study $(87 \% ; n=673)$, followed by TPA $(10 \% ; n=75)$ and transit/combined $(3 \% ; n=24)$ travel modalities. Table 1 outlines the overall respondent profile, which was similar to North Shore City census data. ${ }^{5}$

A total of 75 people engaged in TPA to access their place of work/study, and $50 \%(n=385)$ of all respondents perceived they could access their occupation by TPA modes. Those age $30 \mathrm{y}$ and under were more likely to engage in TPA modes to commute to place of work/study when compared to other age categories, and respondents with trade qualifications were more likely to engage in motorized transport relative to other education classifications. Women were more likely to recognize they could employ TPA modes for commuting to their occupation in comparison to men.

Figure 1 outlines the relationship between commute distance with actual and perceived TPA modes for accessing place of work/study. Participants' perception and actual use of TPA both showed declines as commute distances increased. Table 2 further reveals the likelihood of an individual traveling to work by TPA modes decreasing as commute distance increases.

The group of importance for public health is those who do not use TPA, but perceive they can, therefore we excluded those who engaged in TPA modes for the final analysis. Perusal of Table 3 reveals a strong association between non-TPA mode respondents perceiving they could employ TPA modes to access their occupation and commute distance $(P$-value $<0.001)$. The likelihood of these individuals perceiving they could use TPA modes to access their place of work/study decreased as commute distance increased.

\section{Discussion}

This study confirms that individuals who lived closer to their occupation were more likely to actually, and perceive they could, engage in TPA modes to access their place of work/study. Perception prevalence levels were substantially higher than current TPA engagement. Almost all respondents (97\%) identified they could use TPA modes to access their occupation for distances $1 \mathrm{~km}$ or less, however, less than half $(46 \%)$ of this population currently employed TPA modes for this distance. Steady declines in TPA engagement and perceptions were also shown as commute distance increased. Despite the reduction in actual and perceived TPA engagement with longer distances, when the $5 \mathrm{~km}$ WHO TPA advocacy threshold ${ }^{1}$ is applied to our findings, $47 \%$ of the adult working/studying population commuted within this distance parameter, $68 \%$ of this group perceived they could use, and $18 \%$ actually used, TPA modes for commuting purposes. As such, TPA promotion for up to $5 \mathrm{~km}$ appears to be a reasonable public health recommendation. These findings concur with previous research using an online survey with university staff and students $(n=2210)$. Shannon et al., ${ }^{4}$ reported $30 \%$ of staff and students perceived they could replace their current motorized travel mode to university with TPA means, 


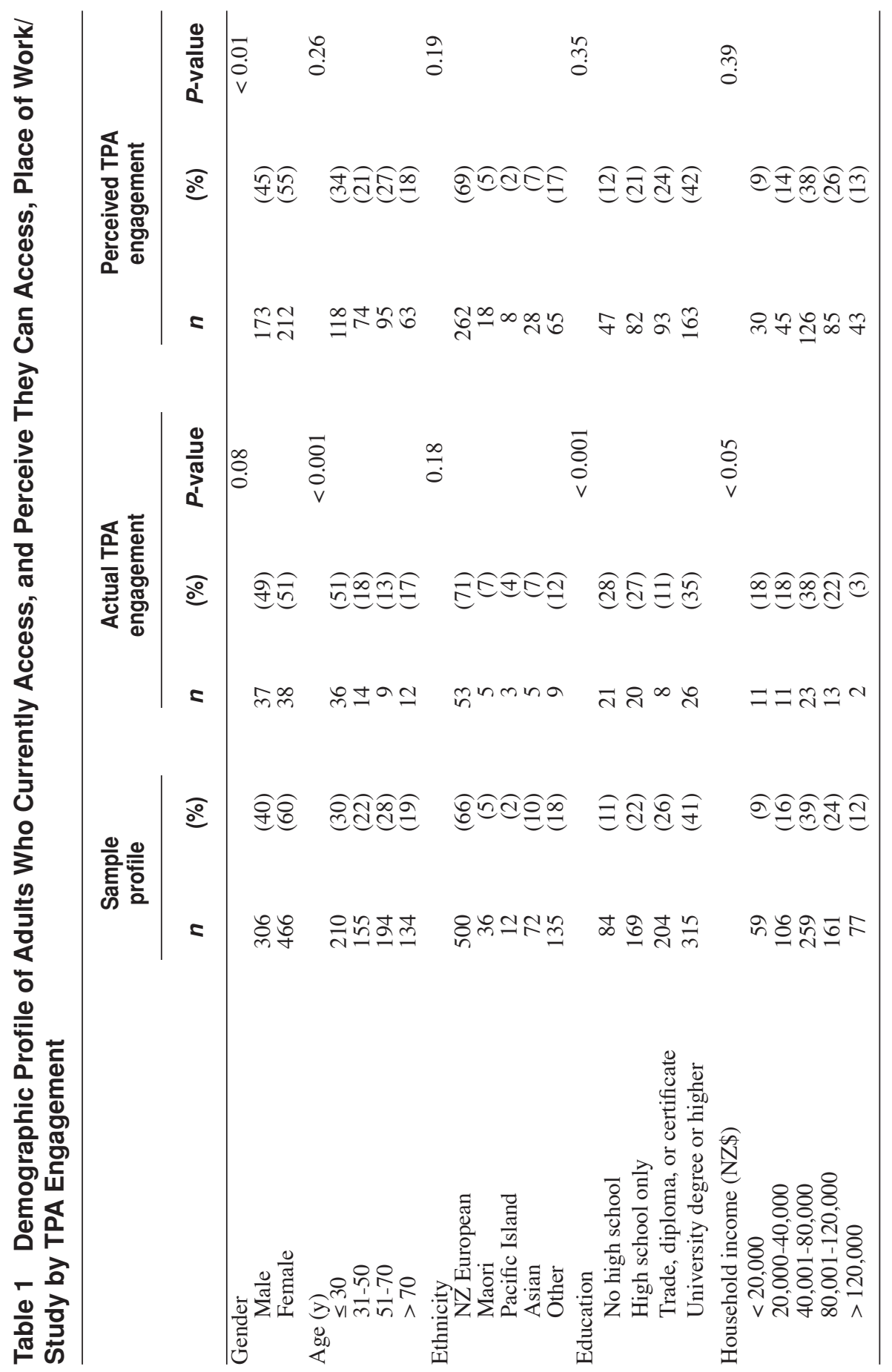




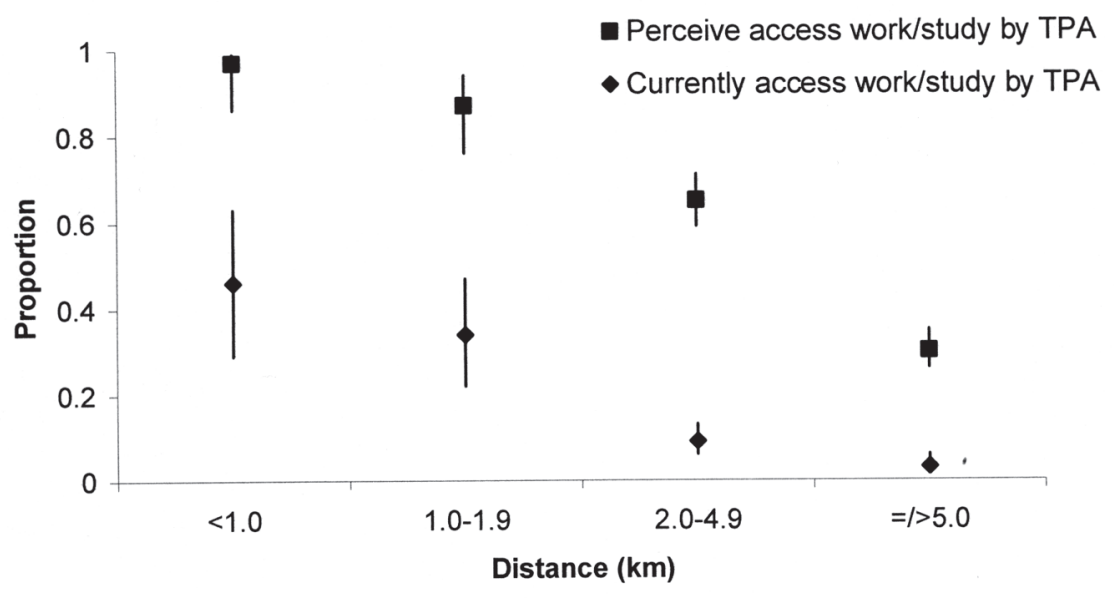

Figure 1-Population proportions of actual and perceived access to place of work/study by TPA modes compared by commute distance.

with a strong relationship between commute distance, time travel, and travel mode evident. Our findings are consistent with other self-reported commute distance and travel modality findings. ${ }^{3}$

Although this study clearly showed the relationships between actual travel modes, perceptions of accessing place of work/study by TPA means, and commute distance, limitations were evident. These include the cross-sectional design, the small sample size, and basing perceptions and travel modalities on self-report. Measuring perceptions of TPA modes is problematic; recognition of TPA participation may not associate strongly with engagement. Nevertheless, perceptions serve to provide a rudimentary indication of TPA acceptance.

\section{Conclusions}

There is considerable recognition of acceptability for TPA modes for distances $5 \mathrm{~km}$ or less. Substantial divergences, however, between travel behaviors and perceptions exist. Prospective work in this field should focus on shifting perceptions to behavior change and developing infrastructure that supports reduced commute distances.

\section{Acknowledgments}

SPARC provided funding for the AFES, and the New Zealand National Heart Foundation supported the first author. Thanks to Nick Garrett and Ed D'Souza for GIS assistance. 

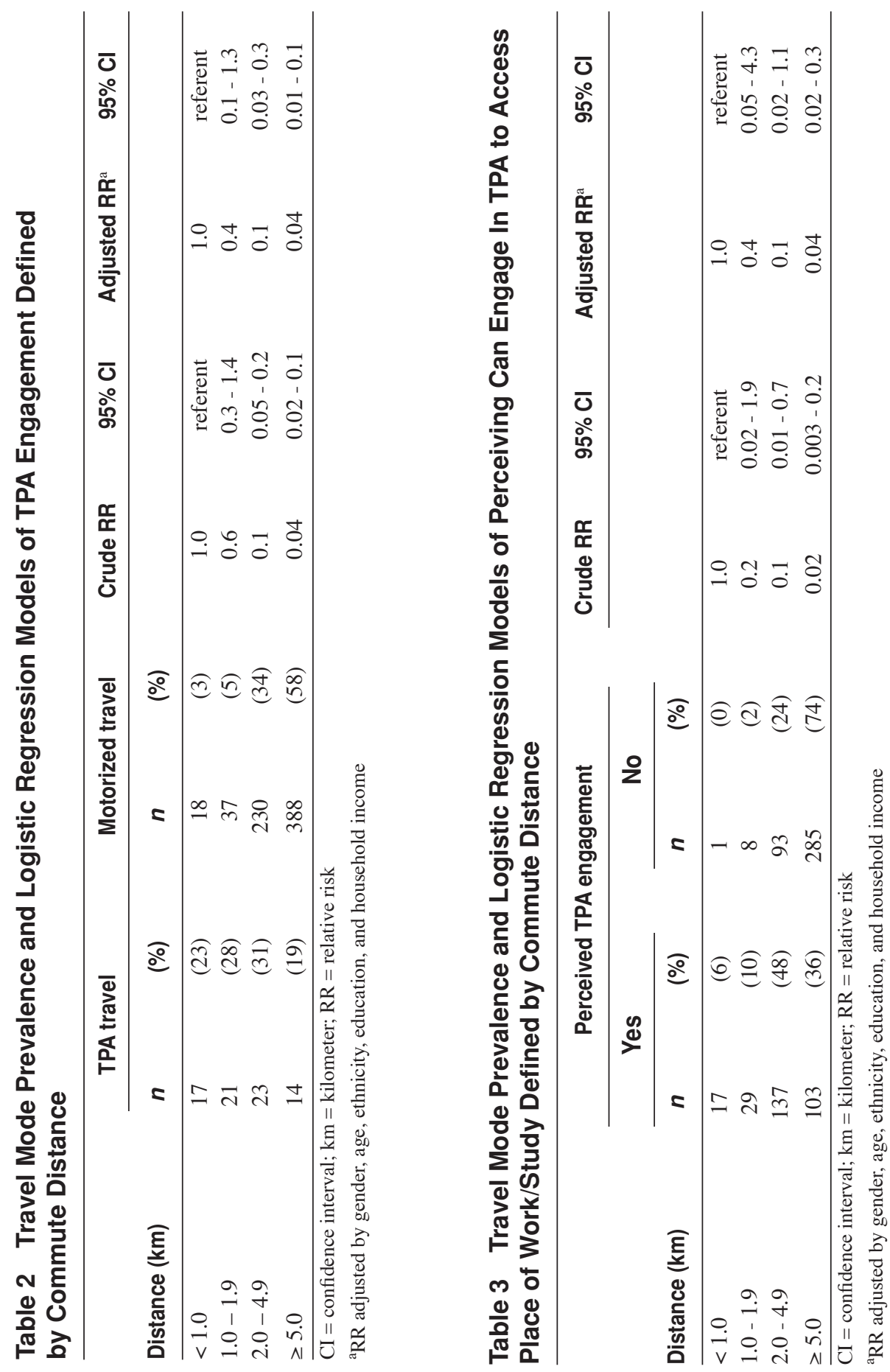


\section{References}

1. World Health Organization. Transport, environment, and health. Austria: Regional Office for Europe of the World Health Organization; 2004.

2. World Health Organization. Global strategy on diet, physical activity, and health. Geneva: World Health Organization; 2004.

3. Plaut PO. Non-motorized commuting in the US. Transport Research Part D: Transport and Environment. 2005;10:347-356.

4. Shannon T, Giles-Corti B, Pikora T, Bulsara M, Shilton R, Bull F. Active commuting in a university setting: Assessing commuting habits and potential for modal change. Transport Policy. 2006;13:240-253.

5. Statistics New Zealand, Census 2001. Wellington: New Zealand Government Statistics Department; 2001. 